\title{
COMPARAÇÃO ENTRE OS DIAGNÓSTICOS CITOPATOLÓGICO E HISTOPATOLÓGICO EM NEOPLASIAS MAMÁRIAS DE CADELAS
}

Recebido: $28 / 02 / 2015$

Aceito:28/01/2016

\author{
RISATI, Ana Carolina ${ }^{1}$; \\ DANEZE, Edmilson Rodrigo ${ }^{2}$; \\ MAGALHÃES, Geórgia Modé ${ }^{3}$.
}

${ }^{1}$ Médica Veterinária Autônoma, Orlândia-SP; ${ }^{2}$ Médico Veterinário, Programa de Pós-graduação em Medicina Veterinária, Faculdade de Ciências Agrárias e Veterinárias, Universidade Estadual Paulista, Jaboticabal-SP; ${ }^{3}$ Médica Veterinária, Docente do Programa de Mestrado em Ciência Animal, Universidade de Franca, Franca-SP.

\section{RESUMO}

objetivo deste estudo foi comparar os resultados obtidos em análises de citopatologia com os de histopatologia no diagnóstico de neoplasias mamárias de cadelas atendidas na rotina do Departamento de Clínica e Cirurgia do Hospital Veterinário da Faculdade Dr. Francisco Maeda (FAFRAM/FE) e da Clínica Veterinária Faro Animal. Num período de seis meses, foi analisado material proveniente de nove cadelas, quatro Terrier Brasileiro, três Poodle e duas sem raça definida. A idade das cadelas variou entre sete e 16 anos. Duas eram castradas e as demais foram castradas durante o procedimento de mastectomia. Uma cadela apresentava três lesões distintas na cadeia mamária, outra, duas lesões, e as demais, uma lesão. Das doze amostras de neoplasias mamárias, sete $(58,3 \%)$ tiveram o diagnóstico sugerido pelos achados do exame citopatológico confirmado no histopatológico, enquanto que em cinco amostras $(41,7 \%)$ não houve confirmação. Os dados deste trabalho permitem concluir que a citopatologia apresentou limitações como técnica diagnóstica nos casos de neoplasias mamárias em cadelas.

Palavras-chave: Cão. Neoplasia. Oncologia. Tumor de mama. 


\section{INTRODUÇÃO}

As neoplasias mamárias são o segundo tipo de neoplasia mais frequentemente observado na rotina de atendimento clínico de cadelas (MACEWEN, 2000; OWEN, 1979; VAIL; STRATMANN et al., 2008), prevalecendo as neoplasias cutâneas primárias (MOULTON et al., 1970; NERURKAR et al., 1989; ZANINOVIC; SIMCIC, 1991). Estima-se que a incidência de malignidade das neoplasias mamárias de cadelas no Brasil seja de 68,4\% (DE NARDI et al., 2002) a 73,4\% (OLIVEIRA-FILHO et al., 2010) dos casos.

O diagnóstico de uma neoplasia mamária em cadelas é obtido pela análise histopatológica da lesão, para caracterização do tipo histológico acometido, grau de malignidade e potencial de invasão do tecido local, além de pesquisa de metástases em linfonodos regionais e locais distantes (CAVALCANTI; CASSALI, 2006; DALECK et al., 2008; FERGUSSON, 1985). Para colher material para análise histopatológica, o paciente pode ser submetido a um procedimento cirúrgico para retirada de um fragmento (biopsia incisional) ou a um procedimento cirúrgico para remoção de toda a lesão, com análise histopatológica posterior (biopsia excisional) (DALECK et al., 2008). No entanto, esses procedimentos podem ser arriscados e desnecessários, pois a lesão pode não ser neoplásica e não necessitar de cirurgia para o tratamento (ARGYLE et al., 2008; COLODEL et al., 2010; MORRIS; DOBSON, 2007; SIMON et al., 2009; THRALL, 2007) ou incompletos, se a lesão for neoplásica e maligna e não for removida com margem suficiente (ALLEN; MAHAFFEY, 1989; DALECK et al., 1998; RISTOW, 2012). Ou, ainda, onerosos ao proprietário, pois se a lesão for neoplásica e o paciente foi submetido apenas à biopsia, ele deverá ser submetido a novo procedimento para exérese total da lesão (ARGYLE et al., 2008; CAMPOS et al., 2011; COLODEL et al., 2010; DALECK et al., 1998; DALECK et al., 2008).

Nesse contexto, a citopatologia apresenta, entre suas aplicações mais importantes, a diferenciação entre um processo inflamatório e uma neoplasia, e uma neoplasia benigna de uma maligna. Sendo, tanto para o clínico quanto para o proprietário, uma opção vantajosa, visto que o procedimento é feito de forma rápida, fácil e com baixo custo, além de ser pouco invasivo quando comparado a outras técnicas de diagnóstico (DALECK et al., 2008; TEIXEIRA et al. 2010; VENTURA et al., 2012). Outra vantagem é a possibilidade de colheita de material 
proveniente de linfonodos, pele ou glândulas sem necessidade de sedação (LAVALLE et al., 2003; ROCHA, 2008).

O objetivo deste estudo foi comparar os resultados obtidos em análises de citopatologia com os de histopatologia no diagnóstico de neoplasias mamárias de cadelas.

\section{MATERIAL E MÉTODOS}

Para esse estudo foram usadas nove cadelas com aumento de volume mamário, sem raça ou idade pré-estabelecidas, atendidas pelo serviço de Clínica e Cirurgia de Pequenos Animais do Hospital Veterinário da Faculdade Dr. Francisco Maeda (HV-FAFRAM/FE) em Ituverava-SP e pela Clínica Veterinária Faro Animal em Orlândia-SP, num período de seis meses.

Durante $o$ atendimento ambulatorial foram obtidas informações referentes a raça, idade e histórico reprodutivo das pacientes. As cadelas com aumento de volume mamário foram submetidas à colheita de material para análise citopatológica e, após mastectomia, amostras de tecido foram encaminhadas para confecção de lâminas histopatológicas.

A colheita de material citológico foi realizada pela técnica da citologia aspirativa por agulha fina (CAAF), realizada em ambulatório. No método de CAAF, fez-se uso de agulha hipodérmica descartável de 25×0,08 mm (BD do Brasil) acoplada à seringa descartável de 20 $\mathrm{mL}$, que foi inserida na lesão previamente tricotomizada e limpa com antisséptico tópico. Realizou-se pressão negativa e, sem retirar a agulha de dentro da massa, reposicionou-se o conjunto com movimentos de vai-vem, descrevendo um leque e tomando amostra de uma pequena área de conteúdo do material. Após, a pressão negativa foi desfeita e a agulha retirada de dentro da massa. Em seguida, a agulha foi desconectada da seringa, que foi preenchida com ar e reconectada à agulha. O conteúdo contido na agulha foi expulso em três lâminas histológicas e, com o auxílio de uma lâmina extensora, o material foi distendido por meio de compressão suave. Os esfregaços obtidos de cada lesão foram então secos ao ar por cinco minutos, fixados e corados com panótico rápido.

Os exames histopatológicos foram realizados através de fragmentos de aproximadamente $1,0 \mathrm{~cm}$ de espessura colhidos da lesão, armazenados em recipientes contendo formol a $10 \%$. Os fragmentos foram processados conforme técnica proposta por Tolosa et al. (2003) e 
corados com Hematoxilina-Eosina. A leitura das lâminas foi padronizada conforme proposto por Cassali et al. (2011) para classificação histológica de neoplasias malignas da glândula mamária canina e o grau de malignidade foi atribuído conforme proposto por Elston e Ellis (1998).

\section{RESULTADOS E DISCUSSÃO}

As raças de cadelas acometidas com neoplasias mamárias foram Terrier Brasileiro (quatro animais), Poodle (três animais) e sem raça definida (dois animais). A idade variou entre sete e 16 anos, sendo que seis animais tinham mais de 10 anos. Duas cadelas eram castradas e as demais foram castradas durante o procedimento de mastectomia.

Uma cadela apresentava três lesões distintas na cadeia mamária, outra, duas lesões, e as demais, uma lesão. Das doze amostras de neoplasias mamárias, sete (58,3\%) tiveram o diagnóstico sugerido pelos achados do exame citopatológico confirmado no histopatológico, enquanto que em cinco amostras $(41,7 \%)$ não houve confirmação.

$\mathrm{Na}$ análise do material citopatológico, em cinco amostras foram observadas apenas células adiposas e/ou células inflamatórias e em sete amostras foram observadas células epiteliais pleomórficas sugestivas de neoplasia (Tabela 1). Na Figura 1, é possível observar duas amostras de análise citopatológica com conteúdo celular sugestivo de carcinoma mamário.

Dentre os casos diagnosticados na histopatologia, as neoplasias de maior frequência foram o carcinoma mamário padrão tubular grau I (três casos), seguido de carcinoma mamário padrão sólido grau III (dois casos) e carcinoma em tumor misto grau I (dois casos), e, em uma amostra, foi identificado condrossarcoma mamário (Tabela 1), que é raro em cadelas (CASSALI et al., 2011). Os animais com carcinoma mamário padrão sólido grau III apresentavam metástase em linfonodo. Na Figura 2, observa-se uma amostra histológica de carcinoma mamário em tumor misto grau I, que apresenta bom prognóstico, e na Figura 3 uma amostra histológica de carcinoma mamário sólido grau III, que apresenta prognóstico reservado. 
Tabela 1 - Comparação entre os diagnósticos citopatológico e histopatológico em neoplasias mamárias de cadelas.

\begin{tabular}{|c|c|c|c|c|}
\hline Animal & Idade & Raça & Diagnóstico citopatológico & Diagnóstico histopatológico \\
\hline 01 & 13 & $\begin{array}{l}\text { Terrier } \\
\text { Brasileiro }\end{array}$ & $\begin{array}{l}\text { M3D - Sugestivo de carcinoma em tumor } \\
\text { misto } \\
\text { M4D - Sugestivo de carcinoma em tumor } \\
\text { misto } \\
\text { M5D - Sugestivo de carcinoma em tumor } \\
\text { misto }\end{array}$ & $\begin{array}{l}\text { M3D - Carcinoma padrão papilar } \\
\text { grau III } \\
\text { M4D - Carcinoma padrão tubular } \\
\text { grau II } \\
\text { M5D - Carcinoma padrão papilar } \\
\text { grau II }\end{array}$ \\
\hline 02 & 13 & $\begin{array}{l}\text { Terrier } \\
\text { Brasileiro }\end{array}$ & $\begin{array}{l}\text { M2D - Não foram visualizadas células } \\
\text { epiteliais. Presença de hemácias e } \\
\text { material protéico. } \\
\text { M4E - Não foram visualizadas células } \\
\text { epiteliais. Presença de hemácias e } \\
\text { material protéico. }\end{array}$ & $\begin{array}{l}\text { M2D - Carcinoma padrão tubular } \\
\text { grau I } \\
\text { M4E - Carcinoma padrão sólido } \\
\text { grau II }\end{array}$ \\
\hline 03 & 7 & Poodle & $\begin{array}{l}\text { Não foram visualizadas células epiteliais. } \\
\text { Presença de material protéico e debris } \\
\text { celulares. }\end{array}$ & Carcinoma padrão tubular grau I \\
\hline 04 & 8 & SRD & $\begin{array}{l}\text { Não foram visualizadas células epiteliais. } \\
\text { Presença acentuada de neutrófilos e } \\
\text { alguns macrófagos. }\end{array}$ & Carcinoma padrão tubular grau I \\
\hline 05 & 10 & $\begin{array}{c}\text { Terrier } \\
\text { Brasileiro }\end{array}$ & Presença de tecido adiposo. & Carcinoma em tumor misto grau I \\
\hline 06 & 16 & SRD & Sugestivo de carcinoma. & Carcinoma em tumor misto grau I \\
\hline 07 & 15 & Poodle & $\begin{array}{l}\text { Presença de células epiteliais com discreto } \\
\text { pleomorfismo, núcleos arredondados, } \\
\text { sem visualização de nucléolos. Presença } \\
\text { de neutrófilos e macrófagos repletos de } \\
\text { gordura em seu citoplasma. }\end{array}$ & Condrossarcoma mamário \\
\hline 08 & 11 & SRD & $\begin{array}{l}\text { Sugestivo de carcinoma de baixo grau de } \\
\text { malignidade. }\end{array}$ & Carcinoma padrão sólido grau III \\
\hline 09 & 12 & $\begin{array}{c}\text { Terrier } \\
\text { Brasileiro }\end{array}$ & Sugestivo de carcinoma. & Carcinoma padrão sólido grau III \\
\hline
\end{tabular}

Legenda: SRD, sem raça definida; $M$, mama; $D$, direita; $E$, esquerda. 


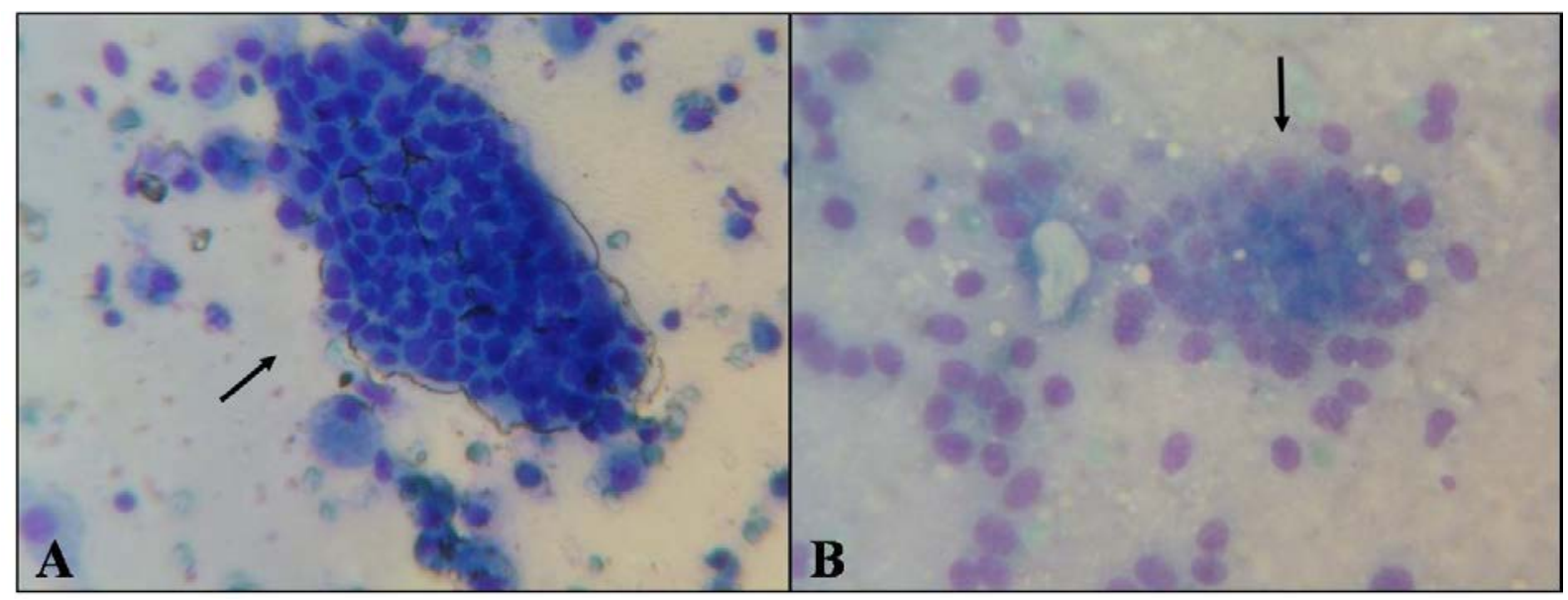

Figura 1 - Fotomicrografia de citologia aspirativa por agulha fina (CAAF) de neoplasia mamária de cadela, panótico, objetiva de 10x. Em A e B, achados sugestivos de carcinoma mamário, notar células epiteliais pleomórficas (setas).

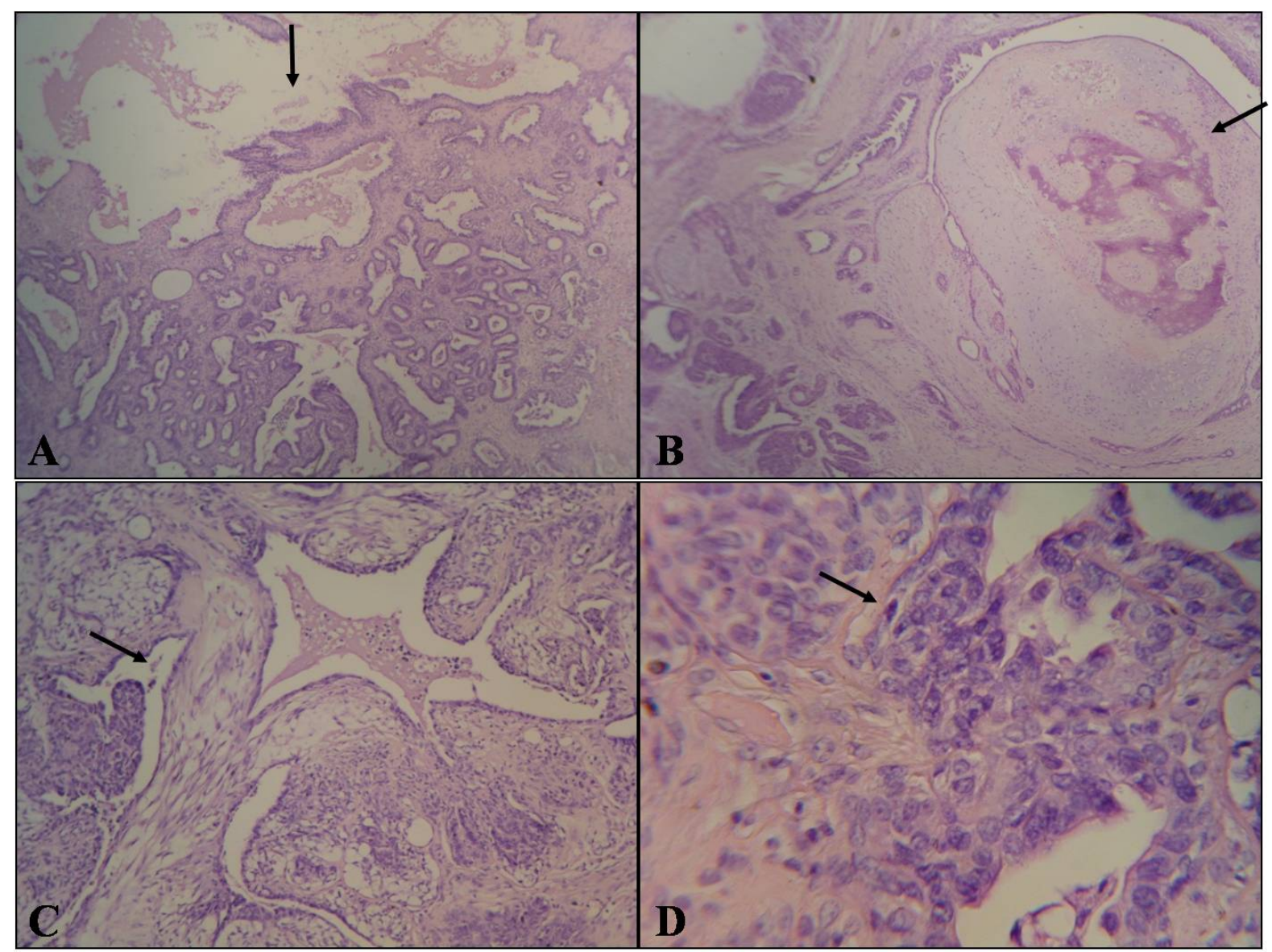

Figura 2 - Fotomicrografia de neoplasia mamária em cadela acometida por carcinoma em tumor misto grau I, H\&E. Em A, notar porções epiteliais e ductos mamários dilatados (seta), objetiva 10x. Em B, notar metaplasia de tecido ósseo (seta), objetiva 10x. Em C, ductos mamários dilatados (seta), objetiva 10x. Em D, notar pleomorfismo celular, objetiva 40x. 


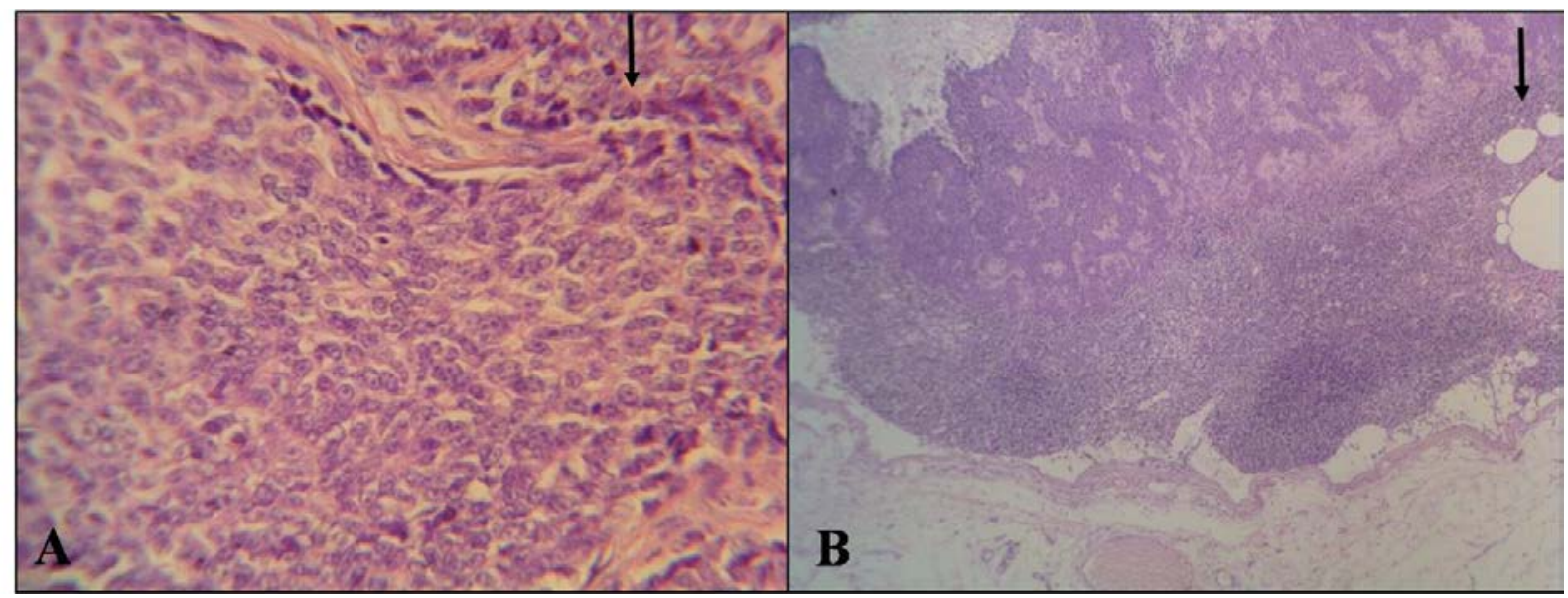

Figura 3 - Fotomicrografia de neoplasia mamária em cadela acometida por carcinoma sólido grau III, H\&E. Em A, notar acentuado pleomorfismo celular (seta), objetiva de 10x. Em B, metástase de carcinoma sólido grau III em linfonodo, observar divisão do linfonodo em tecido normal e tecido neoplásico (seta), objetiva de $4 x$.

As raças acometidas por neoplasias mamárias no presente estudo, Terrier Brasileiro (Fox Paulistinha), Poodle e SRD, fazem parte do grupo de risco para ocorrência de neoplasias mamárias (FURIAN et al., 2007; STONE, 2007; TANAKA, 2003). A incidência é menor em cadelas de raças mestiças ou sem raça definida quando comparadas com as de raças puras (KNAPP et al., 2004). Com relação à idade, verificou-se que todos os animais eram adultos, sendo que seis fêmeas possuíam mais de 10 anos, concordando com a literatura que informa que cadelas adultas apresentam maior incidência da doença (LANA et al., 2007; PELETEIRO, 1994; RUTTEMAN; KIRPENSTEIJN, 2003; STONE, 2007).

Das nove cadelas acompanhadas, duas foram esterilizadas previamente e as demais durante a mastectomia. Segundo a literatura, a ovariohisterectomia (OHE) realizada antes do primeiro estro diminui a incidência de neoplasias mamárias para $0,5 \%$, após o primeiro estro para $8 \%$ e após o segundo estro para $26 \%$, não havendo efeito sobre o desenvolvimento de neoplasias em cadelas castradas após os 30 meses de idade (JONES et al., 2000; MISDORP, 2002; SCHNCIDER et al., 1969; SORENMO et al., 2000), sugerindo que o desenvolvimento das neoplasias mamárias de cadelas pode ser dependente das influências endócrinas do ciclo estral e que a manipulação hormonal pode ser um instrumento favorável em seu tratamento e prevenção (FERGUSSON, 1985; MORRISON, 1998; RUTTEMAN; KIRPENSTEIJN, 2003; SORENMO et al., 2000). 
Em sete $(58,3 \%)$ das doze amostras utilizadas, os diagnósticos citopatológicos e histopatológicos foram coincidentes. Cassali et al. (2006) encontraram um índice de acerto de $67 \%$ e Simon et al. (2009) de $88 \%$ ao comparar uma técnica com a outra. A citologia por aspiração com agulha fina (CAAF) pode indicar se a formação é ou não neoplásica (DALECK et al., 2008; VENTURA et al., 2012), porém nem sempre é fácil a interpretação (COLODEL et al., 2010; MORRIS; DOBSON, 2007). Neoplasias mamárias de grande volume e que apresentam tipos histológicos mistos em geral são de difícil interpretação e diagnóstico no exame citopatológico, contraindicando seu uso nesses casos (COLODEL et al., 2010). No presente estudo, em alguns casos, a variação do tamanho da lesão pode ter prejudicado o exame citológico, como observado na Figura 4.

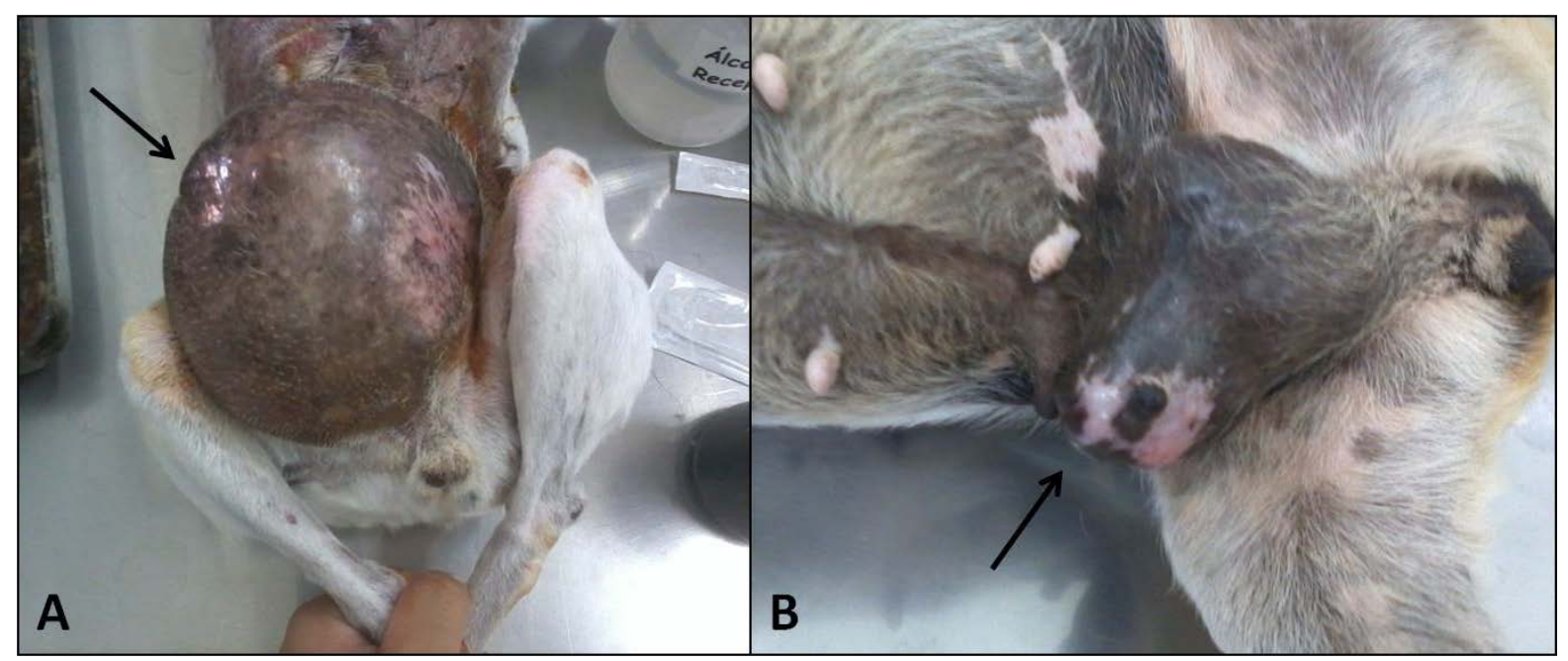

Figura 4 - Neoplasias mamárias de cadelas. Em A, aumento de volume em mama inguinal direita, notar aspecto cístico (seta). Em B, aumento de volume em mama inguinal direita (seta).

Cassali et al. (2011) referem o uso da citologia como método auxiliar com grande utilidade na exclusão de diagnósticos diferenciais como mastite, lipomas e mastocitomas, e que a técnica nunca deve substituir o exame histopatológico no diagnóstico definitivo, pois neoplasias como os carcinomas mamários podem metastizar e causar a morte do animal (GUIM, 2011; PINTO, 2009).

Indica-se a CAAF para acessar linfonodos regionais com suspeita de metástase (ARGYLE et al., 2008; RUTTEMAN; KIRPENSTEIJN, 2003), no entanto, é a biopsia que vai confirmar o diagnóstico, sendo realizada junto com a excisão do nódulo como parte do procedimento de 
tratamento (CASSALI et al., 2011; QUEIROGA; LOPES, 2002), como observado em duas pacientes neste trabalho.

\section{CONCLUSÃO}

Nas condições em que este estudo foi conduzido, concluímos que o diagnóstico do exame citopatológico foi coincidente com o exame histopatológico em 58,3\% dos casos, demonstrando as limitações da citopatologia como técnica diagnóstica nos casos de neoplasias mamárias em cadelas.

\section{COMPARISON BETWEEN CYTOPATHOLOGIC AND HISTOPATHOLOGIC DIAGNOSIS IN BREAST NEOPLASMS OF FEMALE DOGS}

\section{ABSTRACT}

T

he objective of this study was to compare the results obtained in cytopathologic analysis with histopathology in the diagnosis of breast tumors in bitches met in the routine of the Veterinary Hospital's Department of Clinics and Surgery of Dr. Francisco Maeda Faculty (FAFRAM/FE) and Faro Animal Veterinary Clinic. In a period of six months, material from nine female dogs was analyzed, four Brazilian Terriers, three Poodles and two mongrels, the ages varied from seven to 16 years, two were already castrated and the others were castrated during the mastectomy. One bitch had three distinct lesions in the mammary chain; another bitch had two injuries, and the others, one injury each. Of the twelve cases of breast tumors, seven (58.3\%) had the diagnosis suggested by the cytological findings and were confirmed by histopathology, while in five cases $(41.7 \%)$ there was no confirmation. The data of this study showed that the cytopathology presented limitations as a diagnostic technique in cases of breast tumors in female dogs.

Keywords: Dog. Mammary tumor. Neoplasia. Oncology. 


\section{COMPARACIÓN ENTRE LOS DIAGNOSTICOS DE CITOPATOLOGÍA E HISTOPATOLOGÍA EN NEOPLASIAS DE LA MAMA DE PERRAS}

\section{RESUMEN}

$\mathrm{E}$ objetivo de la investigación fue comparar los resultados obtenidos en el análisis de la citopatología con la histopatología en el diagnóstico de neoplasias mamarias en perras asistidas en la rutina del Departamento de Clínica y Cirugía del Hospital Veterinario de la Facultad Dr. Francisco Maeda (FAFRAM/FE) y en la Clínica Veterinaria Faro Animal. Durante un período de seis meses, se analizó material procedente de nueve perras, cuatro Terrier Brasileño, tres Caniche y dos mestizos, con edad entre 7 e 16 años. Dos de esas perras estaban castradas y las otras fueron castrados durante el procedimiento de mastectomía. Una perra tenía tres lesiones distintas en la cadena mamaria, otra, dos lesiones, y las otras, una lesión. De las doce muestras de tumores de mama, siete $(58,3 \%)$ tuvieron el diagnóstico sugerido por los hallazgos del examen citológico confirmado por histopatología, mientras que en cinco muestras $(41,7 \%)$ no hubo una confirmación. Los datos de este estudio mostraron que la citopatología presenta limitaciones como técnica de diagnóstico en los casos de tumores mamarios en perras.

Palabras clave: Perro. Neoplasia. Oncología. Tumor de mama.

\section{REFERÊNCIAS}

ALLEN, S. W.; MAHAFFEY, E. A. Canine mammary neoplasia: prognostic indicators and response to surgical therapy. Journal of the American Animal Hospital Association, v. 25, p. 540-546, 1989.

ARGYLE, D. J.; TUREK, M. M.; MACDONALD, V. Canine and feline mammary tumors. In: ARGYLE, D. J.; BREARLEY, M. J.; TUREK, M. M. Decision making in small animal oncology. Singapura: Wiley-Blackwell, 2008. p. 327-332.

CAMPOS, C. B.; HORTA, R. S.; COBUCCI, G. C.; BOTELHO, F. P. R.; LAVALLE, G. E.; CASSALI, G. $D$. Abordagem cirúrgica das neoplasias mamárias em pequenos animais: perfil do paciente, comportamento e epidemiologia tumoral. Veterinária e Zootecnia, v. 18, n. 2, supl. 2, p. 712, 2011.

CASSALI, G. D.; et al. Consensus for diagnosis, prognosis and treatment of canine mammary tumors. Brazilian Journal of Veterinary Pathology, v. 4, n. 2, p. 153-180, 2011.

CASSALI, G. D.; GOBBI, H.; MALM, C.; SCHMITT, F. C. Evaluation of accuracy of fine needle aspiration cytology for diagnosis of canine mammary tumorus: comparative features with human tumours. Cytopathology, v. 18, p. 191-196, 2006. 
CAVALCANTI, M. F.; CASSALI, G. D. Fatores prognósticos no diagnóstico clínico e histopatológico dos tumores de mama em cadelas-revisão. Clínica Veterinária, v. 11, n. 61, p. 54-64, 2006.

COLODEL, M. M.; RAMOS, R. S.; ROCHA, N. S. Diagnóstico citopatológico de afecções mamárias em cadelas: revisão. Veterinária em Foco, v. 8, n. 1, p. 11-22, 2010.

DALECK, C. R.; FRANCESCHINI, P. H.; ALESSI, A. C.; SANTANA, A. E.; MARTINS, M. I. M. Aspectos clínico e cirúrgicos do tumor mamário canino. Ciência Rural, v. 28, n. 1, p. 95-100, 1998.

DALECK, C. R.; DE NARDI, A. B.; RODASKY, S. Oncologia em cães e gatos. São Paulo: Roca, 2008. p. 75-91, 121-134, 151-160.

DE NARDI, A. B.; RODASKI, S.; SOUSA, R. S.; COSTA, T. A.; MACEDO, T. R.; RODIGHERI, S. M.; RIOS, A.; PIEKARZ, C. H. Prevalência de neoplasias e modalidades de tratamento em cães atendidos no Hospital Veterinário da Universidade Federal do Paraná. Archives of Veterinary Science, v. 7, n. 2, p. 15-26, 2002.

ELSTON, C. W.; ELLIS, I. O. Assessment of histological grade. In: ELSTON, C. W.; ELLIS, I. O. Systemic Pathology. The breast. London: Churchill Livingstone, 1998. p. 365-384.

FERGUSSON, H. R. Canine mammary gland tumors. Veterinary Clinics of North America: Small Animal Practice, v. 15, p. 501-511, 1985.

FURIAN, M.; SANDEI, C. F. C. S.; ROCHA, E. J. N.; LOT, R. F. E. Estudo retrospectivo dos tumores mamários em caninos e felinos atendidos no hospital veterinário da FAMED entre 2003 a 2007. Revista Científica Eletrônica de Medicina Veterinária, v. 4, n. 8, 2007. Dísponivel em:

<http://faef.revista.inf.br/imagens_arquivos/arquivos_destaque/38kobHDxpq7jHSc_2013-521-16-29-17.pdf>. Acesso: 03 fev. 2015.

GUIM, T. N. Determinação de fatores prognósticos para tumores mamários caninos. 2009. 99f. Dissertação (Mestrado Integrado em Medicina Veterinária). Programa de Pós-graduação em Veterinária, Universidade Federal de Pelotas. Pelotas, 2011. Disponível em: <http://repositorio.ufpel.edu.br/handle/123456789/2562>. Acesso: 04 fev. 2015.

JONES, T. C.; HUNT, R. D.; KING, N. W. Patologia veterinária. 6. ed. Barueri: Manole, 2000. p. 1191-1201.

KNAPP, D. W.; WATERS, D. J.; SCHMIDT, B. R. Tumores do sistema urogenital e das glândulas mamárias. In: ETTINGER, S. J.; FELDMAN, E. C. Tratado de medicina interna veterinária: doenças do cão e do gato. 5. ed. Rio de Janeiro: Guanabara Koogan, 2004. 577p. 
LANA, S. E.; RUTTEMAN, G. R.; WITHROW, S. J. Tumors of the mammary gland. In: WITHROW, S. J.; VAIL, D. M. Withrow \& MacEwen's: small animal clinical oncology. 4. ed. St. Louis: Saunders Elsevier, 2007. p. 619-636.

LAVALLE, G. E.; ARAÚJO, R. B.; CARNEIRO, R. A.; PEREIRA, L. C. Punção aspirativa por agulha fina para diagnóstico de mastocitoma em cães. Arquivos Brasileiros de Veterinária e Zootecnia, v. 55, n. 4, p. 500-502, 2003.

MISDORP, W. Tumors of the mammary gland. In: MEUTEN, D. J. Tumors in domestic animals. 4. ed. Ames: lowa State Press, 2002. p. 575-606.

MORRIS, J.; DOBSON, J. Glândula mamária. In: MORRIS, J.; DOBSON, J. Oncologia de pequenos animais. São Paulo: Roca, 2007.

MORRISON, W. B. Canine and feline mammary tumors. In: MORRISON, W. B. Cancer in dogs and cats. Malvern: Williams \& Wilkins, 1998. p. 591-597.

MOULTON, J. E.; TAYLOR, D. D. N.; ANDERSEN, A. G. Canine mammary tumors. Veterinary Pathology, v. 7, n. 4, p. 289-320, 1970.

NERURKAR, V. R.; CHITALE, A. R.; JALNAPURKAR, B. V.; NAIK, S. N.; LALITHA, V. S. Comparative pathology off canine mammary tumors. Journal of Comparative Pathology, $v$. 101, p. 388-397, 1989.

OLIVEIRA FILHO, J. C.; KOMMERS, G. D.; MASUDA, E. K.; MARQUES, B. M. F. P. P.; FIGHERA, R. A.; IRIGOYEN, L. F.; BARROS, C. S. L. Estudo retrospectivo de 1.647 tumores mamários em cães. Pesquisa Veterinária Brasileira, v. 30, n. 2, p. 177-185, 2010.

OWEN, L. N. A comparative study of canine and human breast cancer. Investigative \& Cell Pathology, v. 2, n. 4, p. 257-275, 1979.

PELETEIRO, M. C. Tumores mamários na cadela e na gata. Revista Portuguesa de Ciências Veterinárias, v. 89, n. 509, p. 10-29, 1994.

PINTO, R. M. M. O. Neoplasias mamárias em cadelas e gatas. 2009. 99f. Dissertação (Mestrado Integrado em Medicina Veterinária). Faculdade de Medicina Veterinária, Universidade Técnica de Lisboa. Lisboa, 2009. Disponível em:

<http://www.repository.utl.pt/handle/10400.5/1159>. Acesso: 04 fev. 2015.

QUEIROGA, F; LOPES, C. Tumores mamários caninos: pesquisa de novos fatores de prognóstico. Revista Portuguesa de Ciências Veterinárias, v. 47, n. 543, p. 119-127, 2002.

RISTOW, L. E. Diagnóstico de tumores de mama. Nosso Clínico, v. 15, n. 87, 2012. Disponível em: <http://www.nossoclinico.com.br/Diagnostico-de-Tumores-de-Mama---Edicaon\%C2\%BA-87/8/dicas-laboratorio.aspx>. Acesso: 09 fev. 2015. 
ROCHA, N. S. Exame citológico no diagnóstico de lesões da pele e subcutâneo. Clínica Veterinária, n. 76, p. 76-80, 2008.

RUTTEMAN, G. R.; KIRPENSTEIJN, J. Tumours of the mammary glands. In: DOBSON, J. M.; LASCELLES, B. D. X. BSAVA: Manual of canine and feline oncology. 2. ed. Gloucester: British Small Animal Veterinary Association, 2003. p. 234-242.

SCHNCIDER, R.; DORN, C. R.; TAYLOR, D. O. N. Factors influencing mammary cancer development and postsurgical survival. Journal of the National Cancer Institute, v. 43, p. 1249-1261, 1969.

SIMON, D.; SCHOENROCK, D.; NOLTE, I.; BAUMGÄRTNER, W.; BARRON, R.; MISCHKE, R. Cytologic examination of fine-needle aspirates from mammary gland tumors in the dog: diagnostic accuracy with comparison to histopathology and association with postoperative outcome. Veterinary Clinical Pathology, v. 38, n. 4, p. 521-528, 2009.

SORENMO, K. U.; SHOFER, F. S.; GOLDSCHMIDT M. H. Effect of spaying and timing of spaying on survival of dogs with mammary carcinoma. Journal of Veterinary Internal Medicine, $v$. 14, n. 3, p. 266-270, 2000.

STONE, E. A. Sistema reprodutivo. In: SLATTER, D. Manual de cirurgia de pequenos animais. 3. ed. São Paulo: Manole, 2007. p. 1487-1502.

STRATMANN, N.; FAILING, K.; RICHTER, A.; WEHREND, A. Mammary tumor recurrence in bitches after regional mastectomy. Veterinary Surgery, v. 37, p. 82-86, 2008.

TANAKA, N. Tumor de mama: Qual a melhor conduta? Boletim Informativo da ANCLIVEPASP, v. 7, n. 29, p. 6-7, 2003.

TEIXEIRA, L. V.; LOPES, S. T. A.; MARTINS, D. B.; FRANÇA, R. T.; FIGHERA, R. A. Punção aspirativa por agulha fina como método de coleta de material para a histopatologia no osteossarcoma canino. Pesquisa Veterinária Brasileira, v. 30, p. 132-138, 2010.

THRALL, M. A. Diagnostic cytology in clinical oncology. In: WITHROW, S. J.; VAIL, D. M. Withrow \& MacEwen's: small animal clinical oncology. 4. ed. St. Louis: Saunders Elsevier, 2007. p. 112-133.

TOLOSA, E. M. C.; RODRIGUES, C. J.; BEHMER, O. A.; FREITAS JUNIOR, A. G. Manual de técnicas para histologia normal e patológica. Barueri: Manole, 2003. 341p.

VAIL, D. M.; MACEWEN, E. G. Spontaneously occurring tumors of companion animals as model for human cancer. Cancer Investigation, v. 18, n. 8, p. 781-792, 2000. 
VENTURA, R. F. A.; COLODEL, M. M.; ROCHA, N. S. Exame citológico em medicina veterinária: estudo retrospectivo de 11.468 casos (1994-2008). Pesquisa Veterinária Brasileira, v. 32, n. 11, p. 1169-1173, 2012.

ZANINOVIC, P., SIMCIC, V. Z. B. Epidemiology of mammary tumours in dogs. Veterinaria Fakultaten Universite Ljubljana, v. 28, p. 57-72, 1991.

Autor para correspondência: Edmilson Rodrigo Daneze. Departamento de Clínica e Cirurgia Veterinária, Faculdade de Ciências Agrárias e Veterinárias, Universidade Estadual Paulista. Via de Acesso Prof. Paulo Donato Castellane s/n, CEP 14884-900, Jaboticabal-SP. eddaneze@hotmail.com 